\title{
A Parallel Framework for Fast Photomosaics
}

\author{
Dongwann KANG $^{\dagger a)}$, Member, Sang-Hyun SEO ${ }^{\dagger}$, Seung-Taek RYOO ${ }^{\dagger \dagger}$, and Kyung-Hyun YOON ${ }^{\dagger}$, Nonmembers
}

\begin{abstract}
SUMMARY Main bottleneck of photomosaic algorithm is a search for a best matched image. Unlike several techniques which use fast approximation search for increasing the speed, we propose a parallel framework for fast photomosaic using a programmable GPU. This paper suggests a design of vertex structure for a best match searching on each cell of photomosaic grid and shows a texture representation of image database. The shader programs which are used for searching a best match and rendering image tiles into a display are presented. In addition, a simple duplicate reduction and color correction methods are proposed. Our algorithm not only offers dramatic enhancement of speed, but also always guarantees the 'exact' result.

key words: non-photorealistic rendering, photomosaics, GPU
\end{abstract}

\section{Introduction}

A photomosaic is representation of a source image as a mosaic of small image (usually a photograph) tiles which are arranged in a rectangular grid. The original photomosaic technique, due to Silvers [1] is more closely related to collage or halftone than mosaic in traditional art. In general, photomosaic consists of the steps:

1. Divide a source image into rectangular grids.

2. Search for the image in a database which is most matched to each cell of grid.

3. Replace the cell with the best matches.

Finkelstein and Range [2] suggested using grids that were not rectangular (such as hexagons) and a color correction step. Kim and Pellacini [3] further extended the technique to tiles (not grids) of arbitrary shape. Additional ramifications include video mosaics [4] and stack mosaics [5].

Tran [6] suggested the ways of measuring the effectiveness and the cost of a photomosaic technique: the effectiveness is the similarity between a source image and a photomosaic, and the cost is execution time of a photomosaic algorithm. In general, a huge image database is required to produce an effective photomosaic, because the probability of searching an image that matched with the source image rises with the size of the database. However, the search to find similar images in a database is the main bottleneck in photomosaic algorithms. Therefore making the searches

Manuscript received November 26, 2010.

Manuscript revised June 14, 2011.

${ }^{\dagger}$ The authors are with Chung-Ang Univ., Seoul, 156-756 Korea.

${ }^{\dagger}$ The author is with Han-Shin Univ., O-San, Kyung-gi do, 447791 Korea.

a) E-mail: dongwann@cglab.cau.ac.kr

DOI: 10.1587/transinf.E94.D.2036 more efficient is therefore the most important issue in the designing of photomosaic algorithm.

Tran also suggested the variety of selected image tiles as a measure for quality of photomosaic. Variety was measured as the number of images appeared to create a photomosaic. He suggested a method which limits the number of times that an image can be selected as tiles or imposes on the distance between any two copies of image tiles.

Blasi and Petralia [7] decreased the cost (searching time) by using an antipole tree structure [8], which is much faster than linear search. However antipole tree searches are approximate, so the results may be less effective than slower photomosaic algorithms which search the best matches.

We noticed that photomosaic could be accelerated in the SIMD (Single Instruction, Multiple Data) approach which is a type of parallel processors in Flynn's taxonomy [9], because the searches for each tile can be performed independently. Recent programmable GPUs are the SIMD machines, and they are suitable for the process of photomosaic which renders image tiles on a display. And parallel computing architectures, CUDA, OpenCL, etc which are able to utilize the SIMD machines are suitable for the acceleration of photomosaic process. However, as the searching on each tile (per vertex operation), the blending on each pixel (per fragment operation), and the tile placement (texture mapping) are able to be integrated into rendering pipeline, we use the shader language of GPU.

This paper proposes a novel photomosaic rendering framework which reduces the cost using a parallel process within a GPU. Unlike approximation searching strategy, our goal is reducing the cost without loss of effectiveness. In order to avoid the loss, we employ only exhaustive search to obtain the improvement of cost in the SIMD approach.

\section{Algorithm}

We obtain the cost enhancement by searching the best matched tile which is the bottleneck of photomosaic, using a parallel framework on the SIMD machine (GPU). In this paper, tile matching is performed in the vertex shader of programmable GPU. Therefore designing vertices to correspond to each cell of grid and designing a texture in order to deliver an image database to shader are important issues. These are presented in Sect. 2.1 and 2.2.

The overall framework of our photomosaic algorithm is shown in Fig. 1. The vertex shader searches best matched tiles using exhaustive search. It considers each cell of grid 


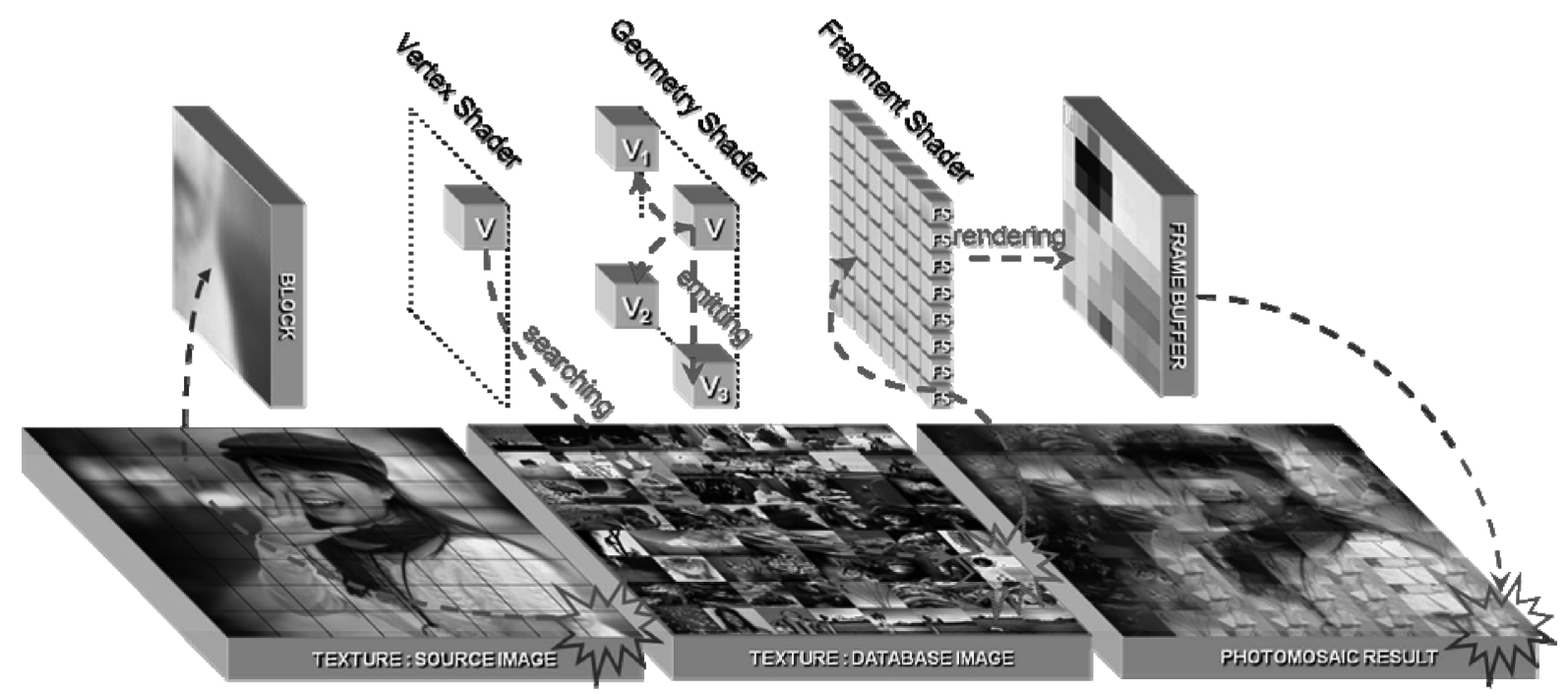

Fig. 1 Our photomosaic generation framework: The vertex shader searches a best matched image in the texture which holds the image database. Then the geometry shader receives the texture coordinate of the best matched image from the vertex shader, and emits three neighbor vertices to make a tile structure. The fragment shader renders the best matched image on the frame buffer.

correspondence to input vertices, and searches for the most similar image in the texture which holds the image database (Sect. 2.3). Then the geometry shader receives the texture coordinate of the best matched image from the vertex shader, and emits three neighbor vertices to make a tile structure (Sect. 2.4). The fragment shader draws the corresponding texels of that tile on the frame buffer (Sect. 2.5). In these step, we suggest a simple duplicate reduction technique which prevents a tile from selecting too many times in image database and a color correction method for enhancing effectiveness.

\subsection{Texture Representation}

Our algorithm should access to two types of image: One is a source image, the other is an image database. The texture is loaded with a source image, and is accessed from the shader programs. However the whole database cannot be loaded into the texture, because current GPUs do not support for hundreds of separate textures. Therefore the database has to be loaded into a limited number of textures, each of limited size.

Loading a texture with actual size of images in database causes complex packing problems. Tiles of a photomosaic are usually very small, so the resolution of the images in the database can be reduced to the size of a photomosaic tile. We reduce the resolution of images in database, and normalize the size of them, and assemble them into a texture (Fig. 2). Although recent GPUs do not force the size of a texture to be a power of 2 , it is a desirable size for accuracy.

Because the size of texture memory is restricted according to the graphics hardware, our available database size is dependent to the GPU. Maximum number of image storable in database, $N$ is follow:

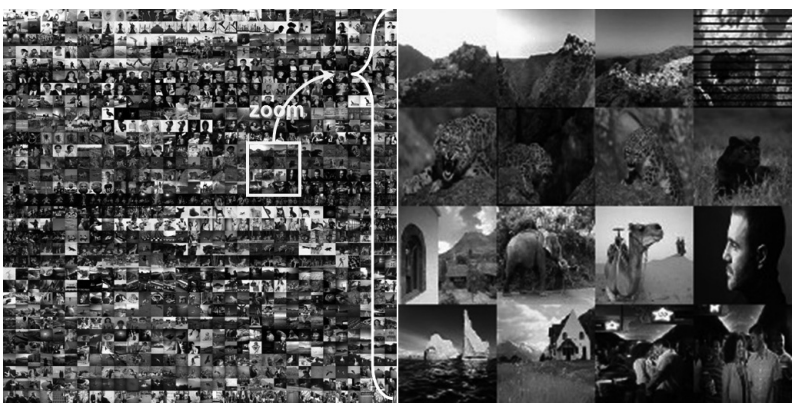

Fig. 2 Image Database Texture: This texture is consists of 1,024 images. All images of database are normalized, and consist the texture.

$$
N=M /(n \times n \times c \times d)
$$

Here, $M$ is the size of available texture memory, $n$ is the size of image, $c$ is the channel of image, and $d$ is the depth of image. As the size of image is increased, the number of image is decreases. Alternatively, as the size of image is decreased, the number of image is increases.

\subsection{Constructing the Vertex Structure}

At least four vertices (at each corner) are required in each tile of photomosaic, in order to construct a photomosaic surface. In this case, general 3D graphics applications may construct the vertex structure that neighboring four tiles share a vertex, in order to prevent the waste of vertex resource. However, this method causes a problem in our algorithm. The texture coordinate of each vertex which is delivered to fragment shader differs from each other, because the best matched image of each tile differs from each other. If four tiles share a vertex, the vertex has to deliver four different texture coordinates to pixels of those tiles. However, it is impossible. 
In order to solve this problem, a vertex structure should not share vertices. If this structure consists of $n$ tiles, $4 n$ vertices are needed. This means that $4 n$ best match searches in the vertex shader are required for $\mathrm{n}$ tiles and duplicated searches occur, because a search for best matched image is performed on the vertex shader. Therefore this is very inefficient.

We render the vertices which correspond to upper-left vertex of each tile using points, not triangles or rectangles. Duplicated searches are eliminated by generating the rest 3 vertices to organize a tile that uses the geometry shader.

The vertex shader requires a piece of a source image which corresponds to each tile. In order to access an appropriate texture coordinate, we generate all texture coordinates of vertices in the range [0:1] for the given axis. 0 is for the minimum value of the coordinate vertex on the axis, and 1 is assigned to the maximum value of the coordinate vertex on the axis.

\subsection{Best Matched Tile Searching Using the Vertex Shader}

When a vertex is taken as an input, first of all, the vertex shader finds a tile of the source image which corresponds to the vertex. We can get the texture coordinate of texels on the tile of the source image, $c$ using texture coordinate of the vertex, $v$, the size of a unit tile, $u$, texel size, $t$ and color of the vertex:

$$
c=\left(v_{x}+t_{x} \cdot i, v_{y}+t_{y} \cdot j\right)(0 \leq i, j<u)
$$

The vertex shader accesses the source image by getting texels of the source image using $c$. In a similar way, the vertex shader accesses the whole image database using the size of tile in the database texture coordinates and the size of a unit texel.

The shader is now ready to look for a tile in the image database that matches the target tile of the source image most closely. In this step, many methods of closest image retrieval are available. For example, the fast multiresolution image querying [10] using wavelet decomposition [11], [12] has been applied to video mosaics [4] which are an extension of photomosaics, and the antipole tree [8] has been used to enhance the speed of photomosaics [7]. However, these are approximate algorithms. Because efficient search of high-dimensional data such as images is very difficult, the effectiveness of these algorithms is relatively low. We therefore do not employ approximate searches. Instead we obtain a best match by using exhaustive search.

We seek the $d_{a b}$ (Eq. (4)) which is the minimum L2 distance from each tile of the source image (a) by accessing the entire database texture (b). The R, G and B in Eq. (3) are the component of color

$$
\begin{aligned}
& c_{i, j}=\sqrt{\left(R_{i, j}^{a}-R_{i, j}^{b}\right)^{2}+\left(G_{i, j}^{a}-G_{i, j}^{b}\right)^{2}+\left(B_{i, j}^{a}-B_{i, j}^{b}\right)^{2}} \\
& d_{a, b}=\sqrt{\sum_{i=1}^{w} \sum_{j=1}^{h}\left(c_{i, j}\right)^{2}}
\end{aligned}
$$

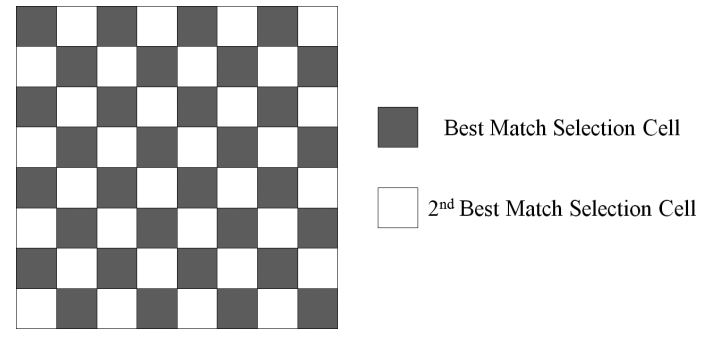

Fig. 3 Simple duplicate reduction: We select the second best match for white tile.

The variety of tile which is proposed by Tran is important in source application, such as photomosaic borders, while the effectiveness is an important factor of best matching search. However we cannot know a best match of neighboring tiles or the number of selection, because we search for a best match in parallel. Therefore, a method that increases the variety of tile is unavailable in this algorithm.

In general, the duplicates around neighboring tiles make photomosaic monotonous. We employ a heuristic strategy which reduces the duplicates on four neighboring tiles. We classify cells of grid into two groups like a chessboard pattern, and let one group select the second best match (Fig. 3). This method cannot guarantee a perfect duplicate reduction, but prevent some duplicates on neighboring tiles.

\subsection{Emitting Vertices using the Geometry Shader}

When the best matched tile is found, we should deliver the texture coordinate of the best match into the fragment shader, and render the tile. However, the rest three vertices are required, since the vertex structure introduced in Sect. 2.2 consists vertices which are the upper-left corner point of each tile.

Using the emitting function of the geometry shader, we generate three duplicates of each vertex which performed the best matching search in Sect. 2.3, move them to each corner of the tile, and construct a mesh. Then we adjust the texture coordinates of the best matched tile of three duplicates to correspond to each corner of the best matched tile.

\subsection{Rendering Tiles Using the Fragment Shader}

The fragment shader completes the photomosaic by rendering the tiles chosen from the image database into the frame buffer. The original photomosaic technique [1] uses the tiles selected from the database without modification, but more recent algorithms include color-correction of each tile from the database, in order to make it more similar to the source image that it is replacing. Finkelstein and Range [2] suggest shifting and scaling the histogram of each tile to match one of the cell more closely. If this method is employed as a post-process, it is possible that there is another tile in the database which matches more with the source image than the currently selected tile after some correction. The entire 
database could be color-corrected before a search, but that is extremely expensive.

We employ a simpler color blending technique based on the following equation:

$$
c_{\text {frame_buffer }}=k \cdot c_{\text {source }}+(1-k) \cdot c_{\text {best_match }}
$$

where $k$ adjusts the blend. User can adjust the blending rate by assigning adequate value for $k$. If $k$ is 0 , the color of the tile from the database is not changed; but if $k$ is 1 , the tile is unchanged from the source image. This method preserves the best match, even if it is performed as a post-process. It can be implemented cheaply on the fragment shader.

\section{Results and Discussion}

We had implemented our algorithm in $\mathrm{C}++$ using the GLSL [13]. Our program requires a Shader Model 3.0 or higher due to requiring conditional branching and loops. We tested our algorithm on a Pentium IV $2.4 \mathrm{GHz}$ machine with 4 GB RAM, NVIDIA GeForce GTX 275 GPU and Microsoft Windows XP.

We compared our results with several other photomosaic algorithms. We measured the effectiveness using the L2 distance which is the square root of the componentwise square of the difference between a source image and the photomosaic result, and cost is defined as execution time in seconds in Tran's study [6]. The competing photomosaic algorithms implemented in $\mathrm{C}++$ are:

1. Exhaustive searching algorithm for minimum $L 2$ dis- tance on a CPU.

2. A fast version of 1 that search for best match using coarse resolution $(4 \times 4)$ tiles.

3. Blasi and Petralia's algorithm [7] which constructs an antipole tree from full-resolution $(16 \times 16)$ tiles.

4. A fast version of 3 using coarse resolution $(4 \times 4)$ tiles.

Figure 4 shows the performance of each algorithm for various sizes of source image. The tiles are $16 \times 16$ and the image database which contains 4,096 images is used. Duplicate reduction and color correction are not applied.

We measure the effectiveness of algorithms using the mean structure similarity (MSSIM) [14] which is the method for assessing image similarity based on perception. The effectiveness of the algorithm 1 which shows the best record is exactly completely same as our result, because we employ the same exhaustive searching algorithm. However, the cost of our result is dramatically reduced, as we employ the SIMD approach. Indubitably the algorithm 4 has the best cost, but the worst effective-ness. The algorithm 3 shows better result in effectiveness than the algorithm 4 , but shows worse result than the algorithm 2 which searches a best match on coarse resolution. These graphs show that the cost of our algorithm is relatively low and the effectiveness is relatively high. However, the variety of exact search, such as algorithm 1 and ours, is relatively lower than other methods.

Figure 5 shows the relation between performance and the size of the image database, for $16 \times 16$ tiles and a $512 \times 512$ source image. Duplicate reduction and color cor-
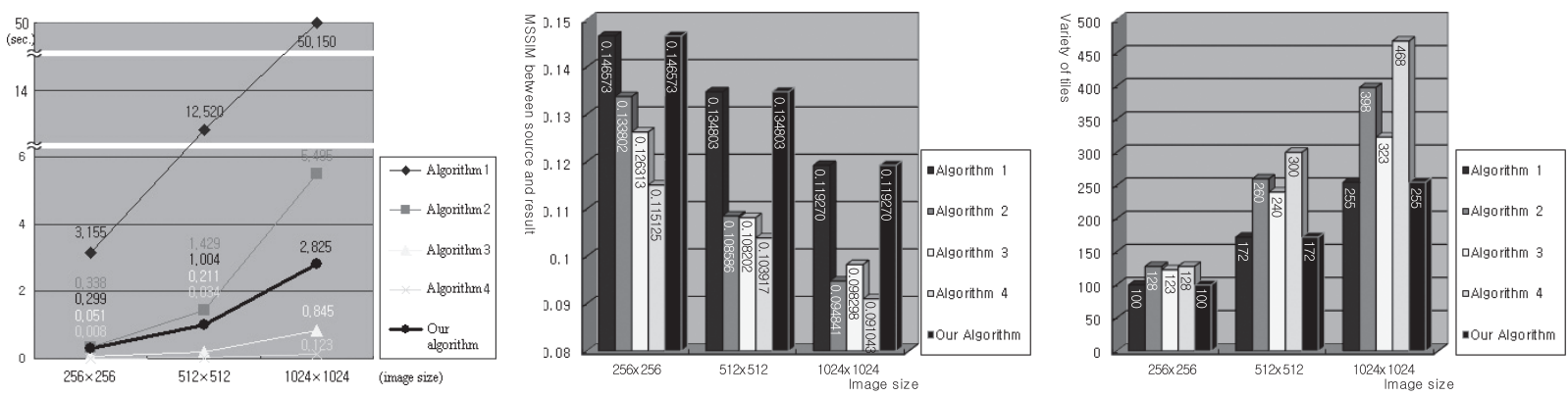

Fig. 4 Performance of each algorithm for various sizes of source image: 4,096 database and $16 \times 16$ tiles are used.
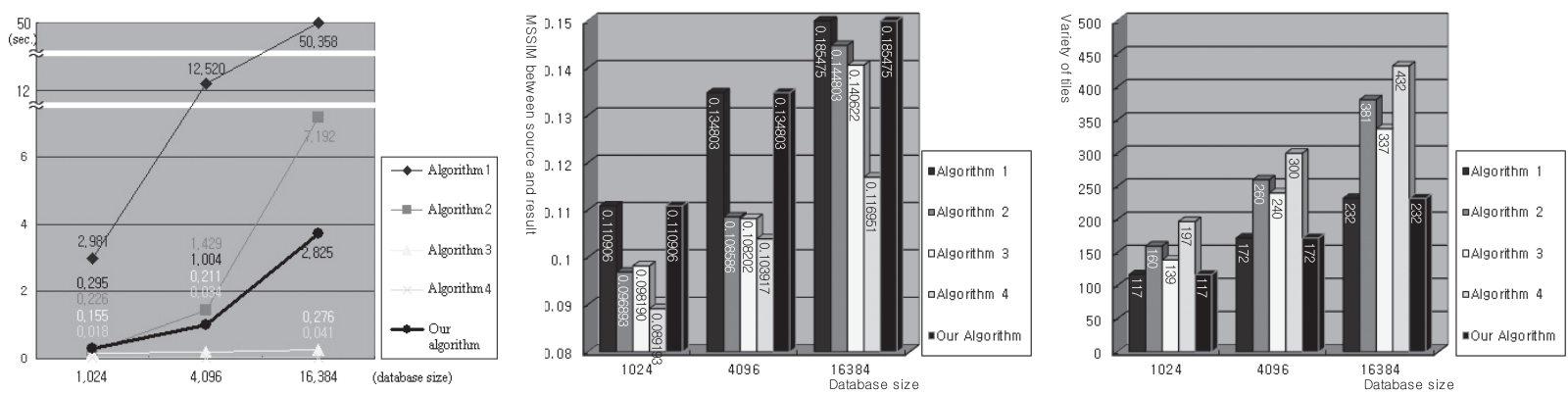

Fig. 5 Performance of each algorithm for various sizes of database: $512 \times 512$ source image and $16 \times 16$ tiles are used. 
rection are not applied. Increase of the database size raises the cost, the quality of matching, and also the variety of tiles. Our algorithm provides the best matching results with much lower cost than the algorithm 1 . The costs of both algorithms are similar when the database size is relative small, but our algorithm is more cost effective when the database size increases than the Algorithm 1. Figure 6 shows the photomosaics of each algorithm in Fig. 5, produced using 16,384 images.

Photomosaic is an algorithm which treats not a movie but an image. Therefore, we suppose that the cost lower than interactive time is enough to perform the algorithm.

Based on this assumption, we provide a new criterion, the score, which evaluates the performance of photomosaic by combining the effectiveness and the cost.

$$
\text { score }=\text { effectiveness } / f^{\text {cost-cost_threshold }}
$$

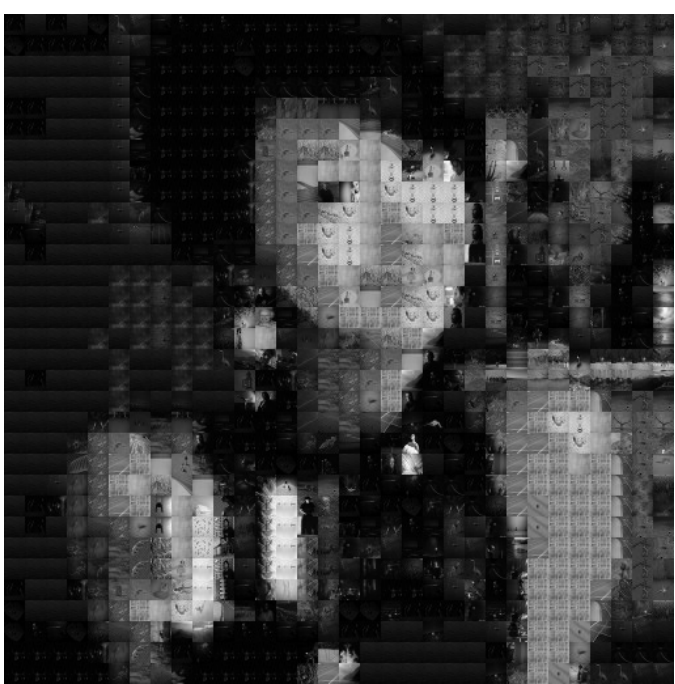

(a) The result of our algorithm and the algorithm1

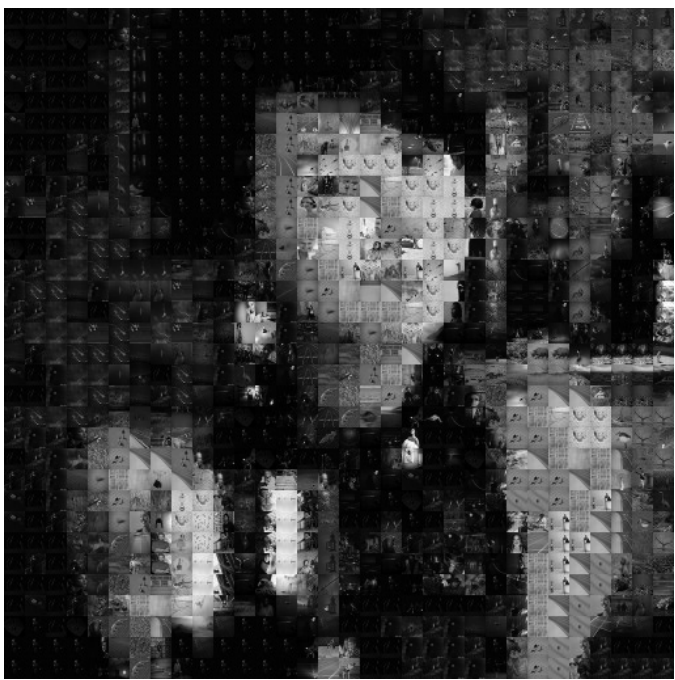

(c) The result of the algorithm3
Here, cost_threshold is a threshold value which defines the border line of interactive time, and $f$ means increasing rate of over cost. We typically use a value of 1.2 for $f$, and a value of 2 for cost_threshold. Figure 7 shows the relation between the score and image size and database size. As shown in figure, our algorithm makes good performance.

Figure 8 shows the effect of duplicate reduction with our algorithm. The variety of Fig. 8 (b) is 238, whereas the variety of Fig. 8 (a) is 304 . Although this method is approximate, it produces reasonably good results. Figure 9 shows the effect after color correction. The result becomes more similar to the source image as the value of $k$ increases, yet it eventually looks contrived.

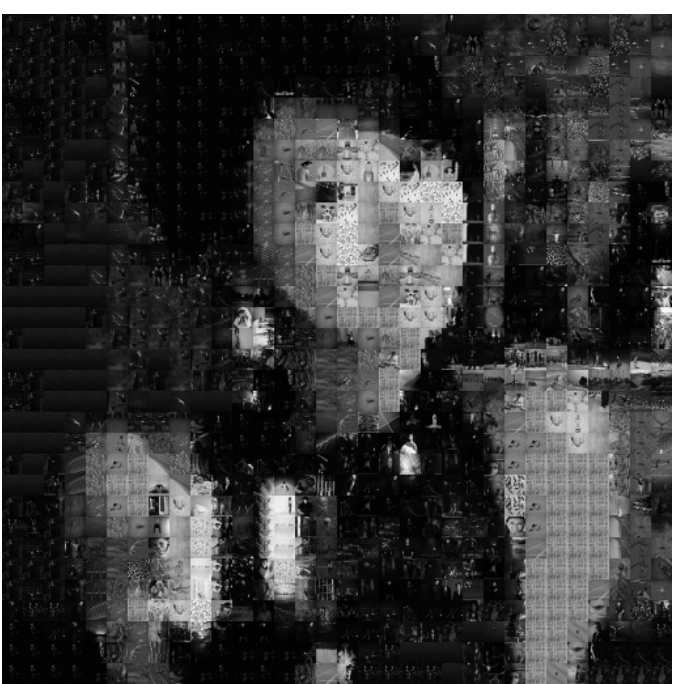

(b) The result of the algorithm2

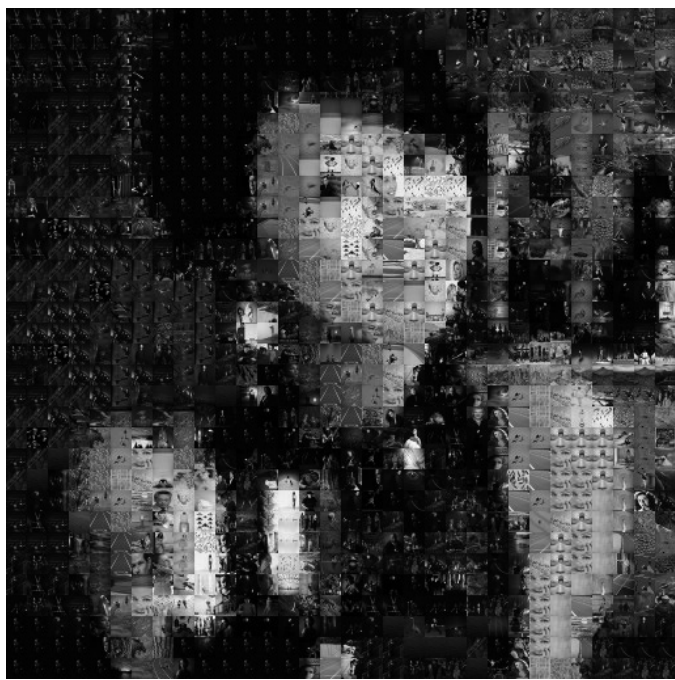

(d) The result of the algorithm4

Fig. 6 Photomosaic image of each algorithm: 16,384 database, $16 \times 16$ tiles and a $512 \times 512$ source image are used. Duplicate reduction and color correction are not applied. 


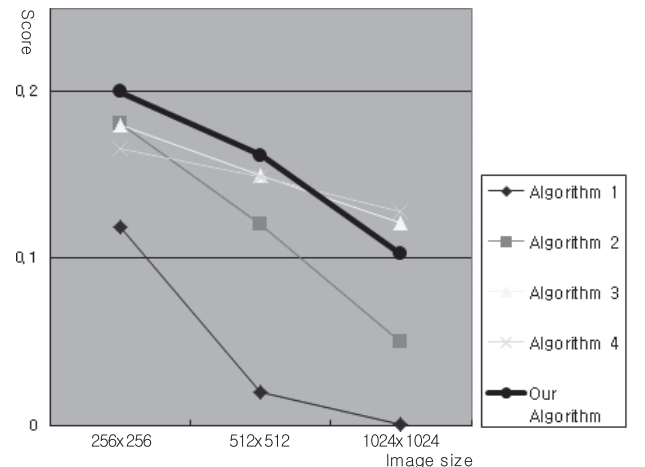

(a) A result without the duplicate reduction

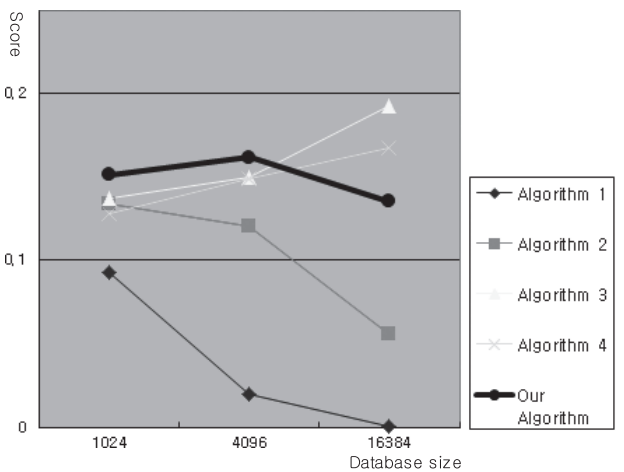

(b) A result with the duplicate reduction

Fig. 7 The score: A new evaluation factor of the performance of photomosaic.

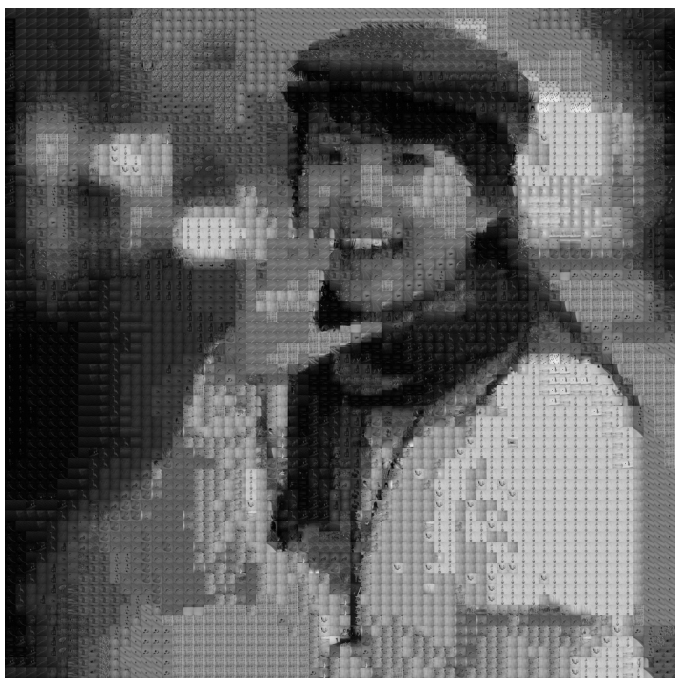

(a) A result without the duplicate reduction

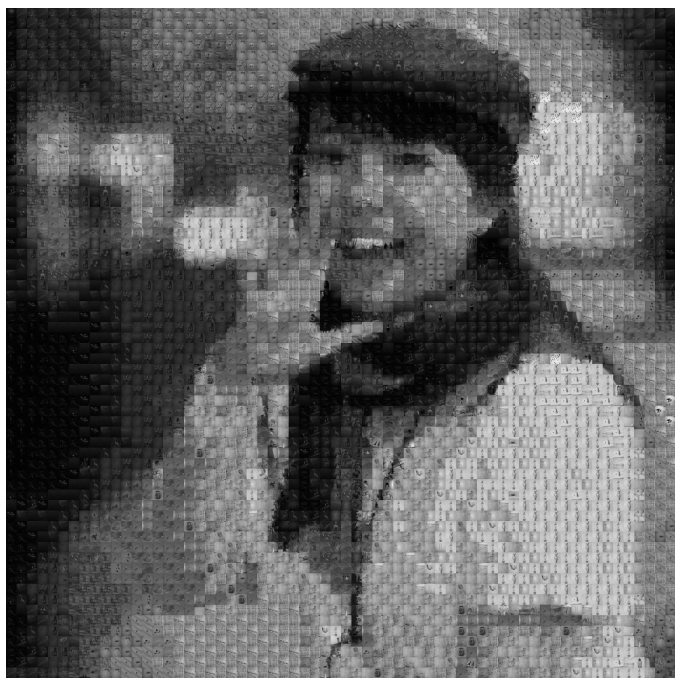

(b) A result with the duplicate reduction

Fig. 8 The effect of duplicate reduction: $16 \times 16$ tiles, $1,024 \times 1,024$ image and database contains 16,384 images are used.

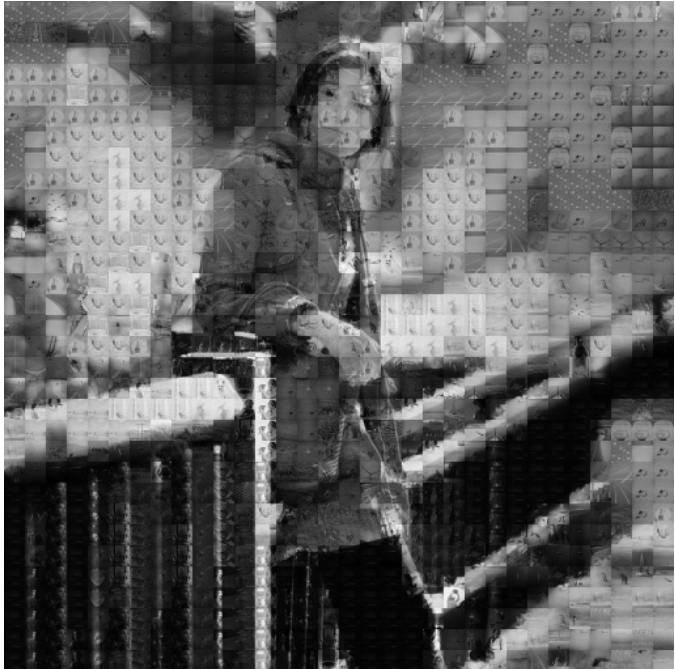

(a) $k=0.0$

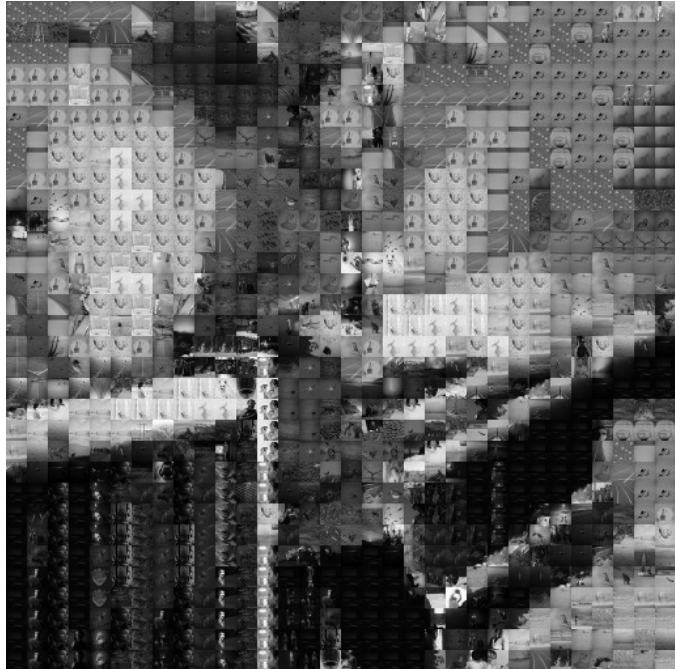

(b) $k=0.33$

Fig. 9 The effect of color correction: $16 \times 16$ tiles, $512 \times 512$ image and database contains 4,096 images are used. 


\section{Conclusions}

By performing tile matching in parallel, using a GPU, we can produce photomosaics much faster than other algorithms while it guarantees the best match searching. Although, our algorithm is not faster than approximate algorithm [7] which employs the antipole search tree, it offers good balance between the cost and the effectiveness. Also, we suggest a simple duplicate reduction method. Though it is coarse method, the result is not so poor for practical uses. Proposed color correction is very fast and creates nice results, although the results looks contrived sometimes.

We find a best match using exhaustive search in this paper, but it is expensive even if it used the SIMD approach. It might be also possible to implement high-dimensional exact search methods in the GPU, which could be expected to produce a photomosaics very quickly indeed.

\section{Acknowledgments}

This work was supported by the Industrial Strategic technology development program, 10035538, developing a tool converting a video to non-photorealistic animations funded by the Ministry of Knowledge Economy (MKE, Korea) and by the National Research Foundation of Korea (NRF) grant funded by the Korea government (MEST) (No.20100018445).

\section{References}

[1] R. Silvers and M. Hawley, Photomosaics, Henry Holt and Co., New York, NY, USA, 1997.

[2] A. Finkelstein and M. Range, "Image mosaics," EP '98/RIDT '98: Proc. 7th International Conference on Electronic Publishing, Held Jointly with the 4th International Conference on Raster Imaging and Digital Typography, pp.11-22, 1998.

[3] J. Kim and F. Pellacini, "Jigsaw image mosaics," SIGGRAPH '02: Proc. 29th Annual Conference on Computer Graphics and Interactive Techniques, pp.657-664, 2002.

[4] A.W. Klein, T. Grant, A. Finkelstein, and M.F. Cohen, "Video mosaics," in NPAR '02: Proc. 2nd International Symposium on NonPhotorealistic Animation and Rendering, pp.21-28, 2002.

[5] J. Park, K. Yoon, and S. Ryoo, "Multi-layered stack mosaic with rotatable objects," Computer Graphics International, 2006, pp.1223, 2006.

[6] N. Tran, "Generating photomosaics: an empirical study," SAC '99: Proc. 1999 ACM Symposium on Applied Computing, pp.105-109, 1999.

[7] G.D. Blasi and P. Maria, "Fast photomosaic," WSCG '05: Poster Proc. 13th International Conference in Central Europe on Computer Graphics, Visualization and Computer Vision'2005, pp.15-16, 2005.

[8] D. Cantone, A. Ferro, A. Pulvirenti, D.R. Recupero, and D. Shasha, "Antipole tree indexing to support range search and k-nearest neighbor search in metric spaces," IEEE Trans. Knowl. Data Eng., vol.17, no.4, pp.535-550, 2005.

[9] M.J. Flynn, "Some computer organizations and their effectiveness," IEEE Trans. Comput., vol.C-21, no.9, pp.948-960, 1972.

[10] E.J. Stollnitz, T.D. DeRose, and D.H. Salesin, "Wavelets for computer graphics: A primer, part 1," IEEE Comput. Graph. Appl., vol.15, no.3, pp.76-84, 1995.
[11] E.J. Stollnitz, T.D. DeRose, and D.H. Salesin, "Wavelets for computer graphics: A primer, part 2," IEEE Comput. Graph. Appl., vol.15, no.4, pp.75-85, 1995.

[12] C.E. Jacobs, A. Finkelstein, and D.H. Salesin, "Fast multiresolution image querying," SIGGRAPH '95: Proc. 22nd Annual Conference on Computer Graphics and Interactive Techniques, pp.277286, 1995.

[13] R.J. Rost, OpenGL(R) Shading Language, 2nd ed., Addison-Wesley Professional, 2006.

[14] Z. Wang, A.C. Bovik, H.R. Sheikh, and E.P. Simoncelli, "Image quality assessment: From error visibility to structural similarity," IEEE Trans. ACM Trans. Image Process., vol.13, no.4, pp.600-612, 2004.

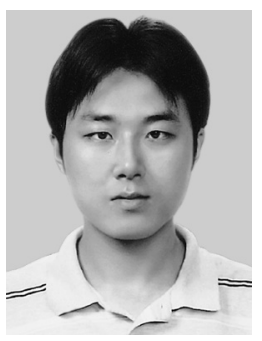

Dongwann Kang is a Ph.D. student in Chung-Ang University, Korea. He received his B.S. and M.S. degrees in Computer Science and Engineering from Chung-Ang University in 2006 and 2008. His research interests include NPR, GPGPU and image processing.

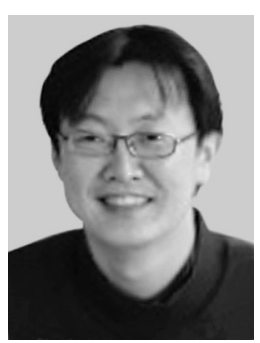

Sang-Hyun Seo received his B.S. degree in Computer Science and Engineering from Chung-Ang Univ., Seoul, Korea, in 1998 and M.S. and Ph.D. degrees in GSAIM at ChungAng Univ. in 2000 and 2010. He was senior researcher at G-Inno System from 2002 to 2005. $\mathrm{He}$ is currently postdoctoral researcher at the University of Lyon 1 in France. His research interests are NPR and 3D GIS system.

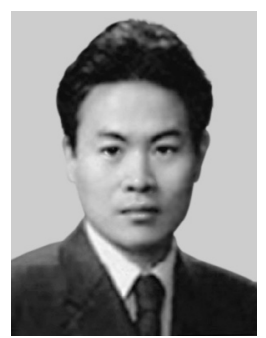

Seung-Taek Ryoo received his B.S. and M.S. degrees in Computer Science and Ph.D. degree in GSAIM Dep at Chung-Ang University in 2002. He is currently a professor at the HanShin University in Korea. His research interests are in the area of GPGPU, Image Based Rendering and Non-photorealistic rendering.

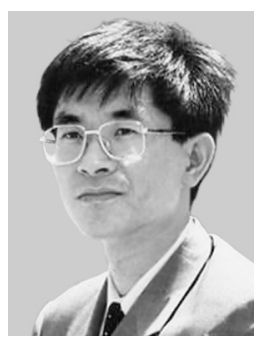

Kyung-Hyun Yoon is a professor of Computer Science and Engineering at Chung-Ang University, Korea. His research interests are in computer graphics and NPR. Prof. Yoon received his B.S. and M.S. degrees from ChunagAng University in 1981 and 1983. He continued his graduate study at University of Connecticut, where he received an M.S. and Ph.D. degrees in Computer Science in 1988 and 1991. He is currently a honorary president of Korean Computer Graphics Society. 